\title{
THE WEAK MIXING ANGLE AND UNIFICATION MASS IN SUPERSYMMETRIC SU(5)
}

\author{
M.B. EINHORN and D.R.T. JONES \\ Randall Laboratory of Physics, University of Michigan, Ann Arbor, MI 48109, USA
}

Received 31 August 1981

\begin{abstract}
We consider the predictions for the weak mixing angle $\theta_{\mathrm{w}}$ and the scale $M$ of unification in a supersymmetric extension of $\mathrm{SU}(5)$, with particular emphasis on the sensitivity to the number of Higgs multiplets. In the one-loop approximation, we also calculate the ratio $m_{\mathbf{b}} / m_{\tau}$. We discuss generally the effects of an intermediate threshold between the weak interaction scale and $M$ and estimate the sensitivity of $\theta_{\mathrm{W}}$ and $M$ to the scale of supersymmetry breaking.

The evolution of the coupling constants of the supersymmetric $\mathrm{SU}(3) \otimes \mathrm{SU}(2) \otimes \mathrm{U}(1)$ effective gauge theory is described and the two-loop corrections to $\theta_{\mathrm{w}}$ and $M$ are calculated.
\end{abstract}

\section{Introduction}

Supersymmetric unified models (SUMs) offer a potential solution to the unnaturalness or gauge hierarchy puzzle of standard grand unified models (GUMs) of electroweak and strong interactions [1,2]. Attempts to build realistic SUMs face theoretical and phenomenological difficulties [2,3], but it has been emphasized [4] that the most naive extension of $\mathrm{SU}(5)$ will not substantially alter the successful prediction of the weak mixing angle $\theta_{\mathrm{w}}$, whereas the unification mass scale $M$ tends to increase by several orders of magnitude, thereby suppressing the proton decay rate beyond the range of feasible observation. Were this a universal feature of SUMs, the observation of proton decay would discourage further attempts to utilize supersymmetry to render GUMs natural ${ }^{\star}$. In this paper, we reconsider the predictions for $\theta_{\mathrm{W}}$ and $M$ in SUMs, emphasizing the sensitivity to the number of light Higgs doublets. We also extend the previous lowest order results to two loops, our motivation being twofold: (i) The accuracy of the one-loop approximation depends upon the size of higher order corrections, and, since SUMs generally will contain many more particles than GUMs, one might a priori expect higher order corrections to be relatively more important. (ii) Even a relatively modest change in the unification mass $M$ produces a large change in the proton lifetime $\tau \propto M^{4}$.

Another topic treated in the course of this investigation is the change in the prediction of the quark-lepton ratio $m_{\mathrm{b}} / m_{\tau}$ in going from the minimal SU(5) GUM to the minimal SU(5) SUM. Remarkably enough, we find only a small change in this seemingly successful consequence of SU(5) unification.

${ }^{\star}$ See note added in proof. 
Another subject of interest which is easily treated in one-loop approximation is the effect of an intermediate threshold between $m_{\mathrm{W}}$ and $M$ on $\theta_{\mathrm{W}}, M$, and the unified coupling constant $\alpha_{\mathrm{G}}$. This can be used in many different applications; for instance, we can deduce the dependence of these parameters on the scale $\Lambda$ of supersymmetric breaking.

The outline of this paper is as follows: In the next section, we review [2-4] the one-loop results for $\theta_{\mathrm{W}}$ and $M$ and discuss the sensitivity to the scale $\Lambda$ of supersymmetry breaking. We also consider the ratio $m_{\mathrm{b}} / m_{\tau}$. In sect. 3, we proceed to consider two-loop corrections in $\theta_{\mathrm{w}}$ and $M$ in SUMs. An interesting new feature of such considerations is that consistency requires the inclusion of certain Yukawa couplings. Finally, in sect. 4, we summarize our conclusions.

In the remainder of this section, we review the evolution of the coupling constants in an $\mathrm{SU}(3) \otimes \mathrm{SU}(2) \otimes \mathrm{U}(1)$ (effective) gauge theory $[5]^{\star}$. Between any two thresholds, the $\beta$-functions are given by

$$
\left.\beta_{i}=b_{i} \frac{g_{i}^{3}}{16 \pi^{2}}+\sum_{j=1}^{3} b_{i j} \frac{g_{i}^{3} g_{j}^{2}}{\left(16 \pi^{2}\right)^{2}}, \quad \text { (not summed on } i\right)
$$

Here $g_{1}, g_{2}$, and $g_{3}$ denote the coupling constants of the U(1), SU(2), and SU(3) gauge groups, respectively. For the standard model, the coefficients $b_{i}, b_{i j}$ are well known; for convenience, they are reproduced in the appendix for an arbitrary number of generations $N_{\mathrm{g}}$ and Higgs doublets $H$. The most naive supersymmetric extension is simply to add appropriate partners for each elementary field appearing in the standard model ${ }^{\star \star}$. Thus we add (Majorana) gauge fermions to the vector bosons, complex scalars to the chiral fermions of each generation, and chiral fermions to the complex Higgs fields. Supersymmetry for the gauge multiplet then implies both Yukawa couplings of the gauge fermion to the matter fermions (equal to the appropriate gauge coupling) and quartic scalar couplings (equal to the square of the gauge coupling). To two loops, the latter does not contribute, but the Yukawa interaction will contribute to the coefficients $\star b_{i j}$. If we continue to neglect other Yukawa couplings ultimately responsible for the light fermion masses, then the result for the $\beta_{i}$ function can be taken from ref. [6], which leads to the formulae in the appendix. Unlike in the standard model, the SU(2) coupling is already not asymptotically free with only 3 generations but increases slowly. Consequently, in SUMs, the coupling constant $\alpha_{\mathrm{G}}$ at the unification mass will always be larger than $\alpha_{2}$ at the supersymmetry breaking scale $\Lambda$.

$\star$ The $\beta$-function through two-loop order for a general $G_{1} \otimes G_{2}$ theory is given in ref. [6].

$\star$ * Several arguments can be advanced against identifying the usual Higgs doublet as the supersymmetric partner of a chiral lepton doublet. For example, there could then be no Yukawa terms responsible for the mass of the lepton after spontaneous symmetry breaking of weak isospin. Also, when embedded in a SUM such as SU(5), the scalar partners of the charge $-\frac{1}{3}$ quarks (such as d) would mediate baryon-violating transitions (Witten, ref. [2]). Finally, the association of Higgs scalars with particular generations is problematic.

$\star \star$ The $\beta$-function for supersymmetric theories was first given in ref. [7]. 


\section{One-loop results}

In lowest order, the solutions for the running coupling constants are

$$
\frac{1}{\alpha_{i}(\mu)}=\frac{1}{\alpha_{\mathrm{G}}}+b_{i} t,
$$

where $t \equiv(1 / 2 \pi) \ln (M / \mu)$. As usual, we will assume that the electromagnetic coupling constant $\alpha$ and the strong interaction coupling constant $\alpha_{3}$ are given at the weak interaction scale $\mu=m_{\mathrm{W}}$, and we thereby determine the unification mass $M$ and unified coupling $\alpha_{\mathrm{G}}$, and we predict the weak mixing angle $\theta_{\mathrm{W}}$. The standard results for these quantities are [5]

$$
\begin{aligned}
\sin ^{2} \theta_{\mathrm{W}} & =\frac{1}{d}\left[b_{2}-b_{3}+\left(b_{1}-b_{2}\right) C^{2} \frac{\alpha}{\alpha_{3}}\right], \\
t & =\frac{1}{d}\left[\frac{1}{\alpha}-\frac{1+C^{2}}{\alpha_{3}}\right], \\
\frac{1}{\alpha_{\mathrm{G}}} & =\frac{1}{d}\left[-\frac{b_{3}}{\alpha}+\frac{\left(b_{2}+C^{2} b_{1}\right)}{\alpha_{3}}\right],
\end{aligned}
$$

where the denominator $d$ is defined by $d \equiv b_{2}-b_{3}+C^{2}\left(b_{1}-b_{3}\right)^{\star}$. The constant $C$ is determined by the normalization of the U(1) hypercharge generator, which depends on the unified group. (For $S U(5), C=\sqrt{\frac{5}{3}}$.) If we insert the formulae for $b_{i}$ from the appendix, we obtain the expressions given in table 1 for the standard model and for its supersymmetric extension. As emphasized in [4], $\sin ^{2} \theta_{W}$ is a function only of the ratio of differences of slopes $b_{i}$, which, with the neglect of Higgs multiplets, is unchanged hy supersymmetrization. In fact, $\sin ^{2} \theta_{\mathrm{w}}$ is a monotonically increasing function of $H$ for which the standard model and supersymmetric model happen to agree at $H=0$ and $H=\infty$. Nevertheless, the two models disagree significantly for finite $H$, as we have displayed in table $2^{\star \star}$.

The unification mass is even more sensitive to the number of Higgs fields. Note that $M$ monotonically decreases with the number of Higgs multiplets $H$. The standard electroweak $\mathrm{SU}(2) \otimes \mathrm{U}(1)$ model generally assumes only a single Higgs doublet, although in GUMs, additional doublets frequently occur. For instance, in SU(5), one might entertain having a 5 and a 45 .

* Note that both $\sin ^{2} \theta_{\mathrm{W}}$ and $t$ depend only on the differences of the $h_{i}$.

* Throughout the paper, we have taken as inputs the values of $\alpha$ and $\alpha_{3}$ at $m_{\mathrm{W}}$, which we chose to be $\alpha^{-1}=128$ and $\alpha_{3}=0.12$, corresponding to $\Lambda_{\mathrm{QCD}} \approx 0.3 \mathrm{GeV}$. (The unification mass is approximately proportional to $\Lambda_{\mathrm{QCD}}$ for small changes in $\alpha_{3}$.) To obtain the absolute normalization of the unification mass $M$, we have taken [8] $m_{\mathrm{W}}=38.5 \mathrm{GeV} / \sin \theta_{\mathrm{W}}$. 
TABLE 1

Synopsis of one-loop results for the unification mass $M\left[t \equiv(1 / 2 \pi) \ln \left(M / m_{\mathrm{W}}\right)\right]$, unified coupling constant $\alpha_{\mathrm{G}}$, and weak mixing angle $\theta_{\mathrm{W}}$

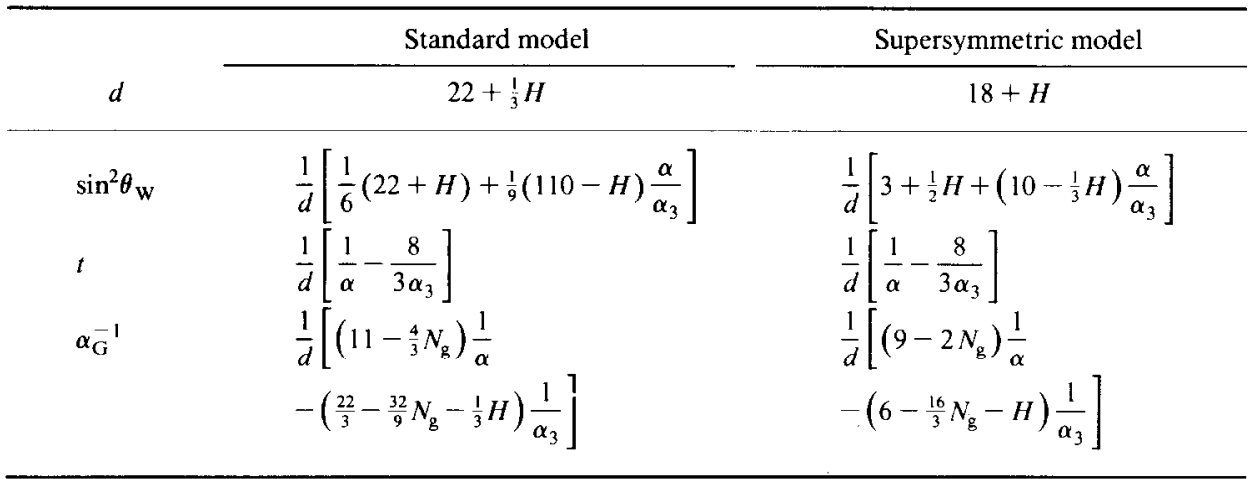

$N_{\mathrm{g}}=$ number of generations; $H=$ number of Higgs doublets.

TABLE 2

Comparison of one-loop results for $\sin ^{2} \theta_{\mathrm{W}}, M$ and $\alpha_{\mathrm{G}}^{-1}$ as a function of the number $H$ of Higgs doublets

\begin{tabular}{cccccccc}
\hline & \multicolumn{3}{c}{ Standard model } & & \multicolumn{3}{c}{ Supersymmetric model } \\
\cline { 2 - 3 } \cline { 7 - 8 } & $\sin ^{2} \theta_{\mathrm{W}}$ & $M\left(10^{14} \mathrm{GeV}\right)$ & $\alpha_{\mathrm{G}}^{-1}$ & & $\sin ^{2} \theta_{\mathrm{W}}$ & $M\left(10^{14} \mathrm{GeV}\right)$ & $\alpha_{\mathrm{G}}{ }^{1}$ \\
\hline$H=0$ & 0.203 & 11 & 42.0 & & 0.203 & 9300 & 26.0 \\
$1^{1}{ }^{\text {a) }}$ & 0.207 & 7.1 & 41.5 & & 0.217 & 1300 & 25.0 \\
2 & 0.211 & 4.5 & 41.0 & & 0.230 & 220 & 24.2 \\
4 & 0.219 & 1.9 & 40.1 & & 0.253 & 10 & 22.8 \\
6 & 0.226 & 0.86 & 39.1 & & 0.272 & 0.78 & 21.6 \\
\hline
\end{tabular}

See 2nd footnote, sect. 2. For $\alpha_{\mathrm{G}}^{-1}$, we have taken $N_{\mathrm{g}}=3$.

a) In the supersymmetric model, the case $H=1$ is necessarily anomalous and included only for comparison.

In table 2, we display the unification mass in one-loop approximation for various numbers of Higgs doublets for both the standard model and its supersymmetric extension. It has been noted $[2,3]$ that a minimum of two Higgs multiplets is necessary in formulating the supersymmetric extension because a single chiral Higgs supermultiplet cannot give mass to both the charge $-\frac{1}{3}$ and charge $+\frac{2}{3}$ quarks. Even more seriously perhaps, a single Higgs supermultiplet would give an anomaly, thereby destroying renormalizability. In fact, to avoid anomalies due to the additional fermions, it is rather natural to double the number of Higgs multiplets. In the non-supersymmetric case, increasing the number of Higgs from 1 to 2 makes only a $35 \%$ decrease in the unification mass; however, in the supersymmetric extension, doubling the number of Higgs from 2 to 4 results in an order of magnitude decrease 


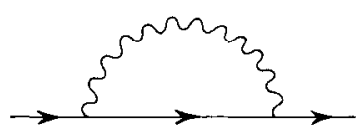

(a)

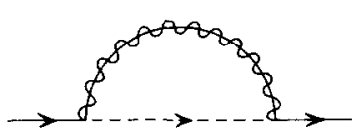

(b)

Fig. 1. Graphs contributing to the one-loop anomalous dimension. Legend: $\rightarrow-=$ chiral fermion:- $\rightarrow-$ $=$ scalar partner of fermion: $n \cap n=$ gauge boson; $\curvearrowleft=$ (Majorana) gauge fermion: (a) vector boson contribution; (b) gauge fermion contribution.

to a value only very slightly greater than the commonly accepted prediction! Thus the observation of proton decay would not ipso facto exclude supersymmetry above 1 $\mathrm{TeV}^{\star}$. However, increasing the number of Higgs multiplets necessarily increases $\sin ^{2} \theta_{\mathrm{W}}$, so it may be difficult for SU(5) SUMs to account simultaneously for both the experimental value of $\sin ^{2} \theta_{\mathrm{W}}$ and an observable proton decay rate. Indeed, all the values of $\sin ^{2} \theta_{\mathrm{W}}$ in table 2 for the supersymmetric case with $H \geqslant 2$ tend to be rather high compared to the currently favored values. [8]

Another frequently cited successful prediction of SU(5) GUMs is the ratio* ${ }^{\star}$ $m_{\mathrm{b}} / m_{\tau}$, which follows from assuming these masses are due to a Higgs $\underset{\sim}{5}$ only. Although this is more controversial and apparently more model dependent than $\sin ^{2} \theta_{\mathrm{W}}$, it is interesting to know how the simplest supersymmetric extension alters the prediction. In addition to the usual self-energy graph (fig. la) contributing to the anomalous dimension of the mass operator $\bar{\psi} \psi$, there is also the graph (fig. 1b) with the gauge fermion and complex scalar partner of the fermion. The general formula for the anomalous dimension $\gamma_{m}$ is changed from

$$
\gamma_{m}=\frac{g^{2}}{16 \pi^{2}} 6 C_{2}(\mathrm{R})
$$

in the standard model to

$$
\gamma_{m}=\frac{g^{2}}{16 \pi^{2}} 4 C_{2}(\mathbf{R})
$$

in the supersymmetric model, where $C_{2}(\mathrm{R})$ is the quadratic Casimir for the representation $\mathrm{R}$ of the matter fields. Then, assuming for simplicity that supersymmetry breaking occurs at $m_{\mathrm{W}}$, we find for the SU(5) supersymmetric model with 3 generations

$$
\frac{m_{\mathrm{b}}}{m_{\tau}}=\left[\frac{\alpha_{3}\left(2 m_{\mathrm{b}}\right)}{\alpha_{3}\left(m_{\mathrm{t}}\right)}\right]^{12 / 23}\left[\frac{\alpha_{3}\left(m_{\mathrm{t}}\right)}{\alpha_{3}\left(m_{\mathrm{W}}\right)}\right]^{4 / 7}\left[\frac{\alpha_{3}\left(m_{\mathrm{W}}\right)}{\alpha_{\mathrm{SUM}}}\right]^{8 / 9},
$$

* Cf. ref. [4]. It is worth recalling [8,9] that there is at lcast an order of magnitude uncertainty in the proton lifetime because of uncertainties in the correct value of $\alpha_{3}$. It has been argued [8] that $H>3$ is excluded because the proton lifetime becomes too short. However, it is clear that many more than 3 doublets are allowed by the supersymmetric extension.

** This has recently been reviewed in ref. [10]. 
while in the standard model

$$
\frac{m_{\mathrm{b}}}{m_{\tau}}=\left[\frac{\alpha_{3}\left(2 m_{\mathrm{b}}\right)}{\alpha_{3}\left(m_{\mathrm{t}}\right)}\right]^{12 / 23}\left[\frac{\alpha_{3}\left(m_{\mathrm{t}}\right)}{\alpha_{\mathrm{GUM}}}\right]^{4 / 7}
$$

Here $m_{\mathrm{t}}$ represents the mass of the top quark. We have neglected the contribution from the hypercharge subgroup, which in both cases gives a correction of $\mathrm{O}(10 \%)$. For $\alpha_{\mathrm{SUM}}=\frac{1}{24}, \alpha_{\mathrm{GUM}}=\frac{1}{41}$ and $m_{\mathrm{t}}=20 \mathrm{GeV}$ we obtain

$$
\left(\frac{m_{\mathrm{b}}}{m_{\mathrm{t}}}\right)_{\mathrm{SUM}}=3.7, \quad\left(\frac{m_{\mathrm{b}}}{m_{\mathrm{t}}}\right)_{\mathrm{GUM}}=3.4
$$

Thus the prediction of the SUM does not differ greatly from that obtained in the standard GUM ${ }^{\star}$. However, in view of the well-known difficulty associated with the mass ratios of the lighter generations of quarks and leptons [10], we would in any case hesitate to describe this as a decisive test of the model.

Another simple exercise is to investigate the effect of an intermediate threshold between $m_{\mathrm{W}}$ and $M^{\star \star}$. The solution of the one-loop equations now becomes

$$
\frac{1}{\alpha_{i}}=\frac{1}{\alpha_{\mathrm{G}}}+\left(b_{i}-B_{i}\right) t+B_{i} T
$$

Here, $2 \pi T \equiv \ln \left(M / m_{\mathrm{W}}\right)$ and $2 \pi t \equiv \ln \left(\Lambda / m_{\mathrm{W}}\right)$, where $\Lambda$ represents the scale at which the intermediate threshold occurs. The slope parameter below (above) the threshold is called $b_{i}\left(B_{i}\right)$. Of course, if the threshold is regarded as known, one can again solve for $\alpha_{\mathrm{G}}, M$, and $\sin ^{2} \theta_{\mathrm{W}}$. We find

$$
\sin ^{2} \theta_{\mathrm{W}}=\frac{1}{D}\left(B_{2}-B_{3}+C^{2}\left(B_{1}-B_{2}\right) \frac{\alpha}{\alpha_{3}}\right)-\frac{\alpha t C^{2}}{D}\left|\begin{array}{ll}
b_{1}-b_{2} & b_{2}-b_{3} \\
B_{1}-B_{2} & B_{2}-B_{3}
\end{array}\right|
$$

where $D \equiv B_{2}-B_{3}+C^{2}\left(B_{1}-B_{3}\right)$. Note that $\sin ^{2} \theta_{\mathrm{w}}$ varies linearly with $t$ between the value in the case where $\Lambda=m_{\mathrm{W}}(t=0)$ to the value when the threshold is at the unification mass, $\Lambda=M(t=T)$. Thus, if these two values lie within the range of experimental uncertainty in $\sin ^{2} \theta_{\mathrm{W}}$, then the presence of an intermediate threshold at an unknown scale does not really vitiate the prediction of the weak mixing angle.

* It must be noted that this ratio was already considered in ref. [3], who quote a different result from ours.

* These considerations are similar in spirit to those of ref. [11]. 
The unification mass and $\alpha_{G}^{-1}$ also depend linearly on $t$, viz.,

$$
\begin{aligned}
T= & \frac{1}{D}\left(\frac{1}{\alpha}-\frac{\left(1+C^{2}\right)}{\alpha_{3}}\right) \\
& +\frac{t}{D}\left[B_{2}-B_{3}-\left(b_{2}-b_{3}\right)+C^{2}\left(B_{1}-B_{3}-\left(b_{1}-b_{3}\right)\right)\right], \\
\frac{1}{\alpha_{G}}= & \frac{1}{D}\left(-\frac{B_{3}}{\alpha}+\frac{\left(B_{2}+C^{2} B_{1}\right)}{\alpha_{3}}\right)+\frac{t}{D}\left|\begin{array}{ll}
h_{2}+C^{2} b_{1} & b_{3} \\
B_{2}+C^{2} B_{1} & B_{3}
\end{array}\right| .
\end{aligned}
$$

These also vary between the values in the two extremes $\Lambda=m_{\mathrm{W}}$ and $\Lambda=M$. So it is trivial to estimate the effect of an intermediate threshold of a known type (new generation, heavy Higgs, etc.) In SU(5), it is a fact [11] that, if all the particles which compose a complete representation of $\mathrm{SU}(5)$ enter at the same mass scale, they contribute equally to all $B_{i}$ and so make no change at all (in the one-loop approximation ${ }^{\star}$ ) in either $\sin ^{2} \theta_{\mathrm{w}}$ or $T$. Thus, the proton lifetime will be affected only via the change in $\alpha_{G}$. On the other hand, "incomplete" multiplets (such as a Higgs doublets without its colored partners) affect the mixing angle and unification mass already at the one-loop level. The preceding formulas can be applied to calculate, for example, the dependence on the scale $\Lambda$ of supersymmetry breaking. For the coefficients given in the appendix, eqs. (2.8) and (2.9) become

$$
\begin{aligned}
\sin ^{2} \theta_{\mathrm{W}} & =\left(\sin ^{2} \theta_{\mathrm{W}}\right)_{0}-\frac{16}{3} \frac{\alpha H}{(18+H)} t, \\
T & =T_{0}-\frac{\left(4-\frac{2}{3} H\right)}{(18+H)} t, \\
\alpha_{\mathrm{G}}^{-1} & =\left(\alpha_{\mathrm{G}}^{-1}\right)_{0}+\frac{t}{18+H}\left(20 N_{\mathrm{g}}+8 H-\frac{2}{3} N_{\mathrm{g}} H\right),
\end{aligned}
$$

where the first term on the right-hand side of each equation is given by the value in the supersymmetric theory assuming $\Lambda=m_{\mathrm{w}}$ (table 1). For example, for $H=2$, $N_{\mathrm{g}}=3$, we get

$$
\begin{aligned}
\sin ^{2} \theta_{\mathrm{W}} & =\left(\sin ^{2} \theta_{W}\right)_{0}-\frac{8}{15} \alpha t \approx 0.230-0.004 t, \\
T & =T_{0}-\frac{2}{15} t \approx 5.29-0.13 t, \\
\alpha_{\mathrm{G}}^{-1} & =\left(\alpha_{\mathrm{G}}^{-1}\right)_{0}+\frac{18}{5} t \approx 24.2+3.6 t,
\end{aligned}
$$

\footnotetext{
* This is not true beyond one-loop. The two-loop effects of a new generation are considered in [9].
} 
so that, per unit of $t=(1 / 2 \pi) \ln \left(\Lambda / m_{\mathrm{W}}\right)$, we find a percentage change in $\sin ^{2} \theta_{\mathrm{W}}, M$, and $\alpha_{G}$ by $-2 \%,-67 \%$, and $-13 \%$, respectively. (For naturalness, we expect $\Lambda \lesssim 1$ $\mathrm{TeV}$ or $t \lesssim 0.4$.) It is also worth emphasizing how small a change this makes in $\sin ^{2} \theta_{\mathrm{w}}$, which leads us to wonder whether the SU(5) prediction of the mixing angle is not much more stable against new thresholds in the desert region than is generally believed.

\section{Two-loop results}

The determination of two-loop corrections to $\theta_{\mathrm{W}}$ and $M$ is quite important. Since the one-loop corrections to $\sin ^{2} \theta_{\mathrm{W}}$ are on the order of $50 \%$, one worries about the rate of convergence of the perturbation expansion and the accuracy of the lowest order result.

The solution of the renormalization group equations through two loops has been discussed by a number of people $[8,9,12]$, so we can adapt their methods of solution to the supersymmetric case. The coefficients $b_{i j}$ needed have already been displayed in the appendix. However, one other piece of information is needed to solve the equations, viz., the "boundary" or "matching" condition relating the coupling constants $\alpha_{i}$ of the $\mathrm{SU}(3) \otimes \mathrm{SU}(2) \otimes \mathrm{U}(1)$ effective gauge theory to the coupling constant $\alpha_{\mathrm{G}}$ of the unified theory in the region near the unification mass: In any gauge theory, the relation is of the form

$$
\begin{aligned}
\frac{1}{\alpha_{i}(\mu)}=\frac{1}{\alpha_{\mathrm{G}}(\mu)}-\frac{1}{2 \pi}\left\{\frac{1}{6} \operatorname{Tr}\left[T_{i \mathrm{~S}}^{2} \Lambda_{\mathrm{S}} \ln \frac{M_{\mathrm{S}}}{\mu}\right]\right. \\
\left.+\frac{4}{3} \operatorname{Tr}\left[T_{i \mathrm{~F}}^{2} \ln \frac{M_{\mathrm{F}}}{\mu}\right]-\frac{7}{2} \operatorname{Tr}\left[T_{i \mathrm{~V}}^{2} \ln \frac{M_{\mathrm{V}}}{\mu}\right]+C_{i}\right\},
\end{aligned}
$$

where $T_{i A}$ represents the generator for scalars $(\mathrm{S})$, fermions $(\mathrm{F})$, and vectors $(\mathrm{V})$. The traces are summed over the superheavy scalars, fermions, and vectors, where $\Lambda_{\mathrm{S}}$ is a projector removing those scalar, would-be Goldstone bosons which were eaten to give massive vectors ${ }^{\star}$. As emphasized by Hall [12], the whole question is what are the integration constants $C_{i}$, since the dependence on the scale $\mu$ is dictated by the lowest order renormalization group equation for $\alpha_{i}$ and $\alpha_{\mathrm{G}}$. In fact, the answer depends on the renormalization scheme used. For example, for modified minimal subtraction, Weinberg [12] gives

$$
C_{i}=\frac{1}{6} \operatorname{Tr} T_{i \mathrm{~V}}^{2}+\frac{4}{3} \operatorname{Tr}\left(T_{i \mathrm{~F}}^{2}\right) \sqrt{2}
$$

* The normalization of each trace has been chosen as would be appropriate for a scalar transforming by a real representation of the group; a Dirac fermion, by a complex representation; a real vector, by a real (i.e. the adjoint) representation. For a complex scalar, multiply by 2 ; for a Majorana fermion. divide by 2 . 
TABIF 3

Two-loop results for the supersymmetric model with 3 generations as a function of the number of Higgs doublets $H$

\begin{tabular}{ccccccc}
\hline$H$ & $\Delta \sin ^{2} \theta_{\mathrm{W}}\left(10^{-3}\right)$ & $\sin ^{2} \theta_{\mathrm{W}}$ & $\Delta \ln \left(M / m_{\mathrm{W}}\right)$ & $M\left(10^{14} \mathrm{GeV}\right)$ & $\Delta \alpha_{\mathrm{G}}^{-1}$ & $\alpha_{\mathrm{G}}^{-1}$ \\
\hline 0 & 2.8 & 0.206 & -0.200 & 7500 & -0.705 & 25.3 \\
2 & 3.0 & 0.233 & -0.223 & 170 & -0.667 & 23.5 \\
4 & 3.2 & 0.256 & -0.232 & 8.0 & -0.633 & 22.2 \\
6 & 3.3 & 0.275 & -0.235 & 0.62 & -0.601 & 21.0 \\
\hline
\end{tabular}

whereas Hall drops the second term. The origin of the constant $C_{i}$ depends on how one continues the fermion and vector boson degrees of freedom away from four dimensions. Within standard models, this is somewhat arbitrary (as the difference between Hall and Weinberg illustrates). However, in supersymmetric theories, one must regulate without altering the spin degrees of freedom in order to preserve supersymmetry ${ }^{\star}$. It is a simple exercise then to show that this requires $C_{i}=0$. Thus, except for superheavy mass differences ${ }^{\star \star}$, the boundary condition for the second-order calculation is the same as in lowest order, viz., $\alpha_{i}(M)=\alpha_{\mathrm{G}}$.

Now it is straightforward to modify previous two-loop solutions to the supersymmetric case. (See in particular Hall, and Unger and Yao [12], who used modified minimal subtraction, which we follow.) In the appendix, we have summarized the two-loop formulae for $\sin ^{2} \theta_{\mathrm{W}}, M$, and $\alpha_{\mathrm{G}}^{-1}$. Having no information on the masses of superheavy particles, we have used the matching condition $\alpha_{i}(M)=\alpha_{\mathrm{G}}$. We display the results in table $3^{\star \star \star}$. (See 2 nd footnote of sect. 2.) We have indicated the results for various numbers of generations and Higgs doublets. In all cases, the two-loop

* A supersymmetric, gauge-invariant regularization has been suggested in ref. [13a] and elaborated further in $[13 \mathrm{~b}]$.

ॠ The crossing of a gauge threshold above the supersymmetry threshold corresponds to the boundary condition

$$
\alpha_{i}(\mu)^{-1}=\alpha(\mu)^{-1}-\frac{1}{\pi}\left[\operatorname{Tr} T_{i \mathrm{H}}^{2} \Lambda_{\mathrm{S}} \ln \frac{M_{\mathrm{H}}}{\mu}+\operatorname{Tr} T_{i \mathrm{~F}}^{2} \ln \frac{M_{\mathrm{F}}}{\mu}-\operatorname{Tr} T_{i \mathrm{~V}}^{2} \ln \frac{M_{\mathrm{V}}}{\mu}\right] .
$$

where the first term in the bracket represents the contribution from massive Higgs multiplets; the second, from massive matter multiplets: the third. for massive vector supermultiplets. (The matter and Higgs multiplets are assumed to consist of a Dirac spinor plus two complex scalars transforming by a complex representation of the group. For a real representation, divide by two; for a Majorana multiplet, divide by two. The massive vector supermultiplet consists of a real vector, a Dirac fermion, and a real scalar, all transforming in the adjoint representation.)

$\star \star \star$ Of course, the equations only determine the ratio $M / m_{\mathrm{w}}$. Strictly speaking, we ought to compute the one-loop corrections to $m_{\mathrm{W}}$ in order to determine the absolute scale for $M$. However, since the uncertainties in $M$ due to uncertainties in $\alpha_{3}$ are large [8,9], this small correction to $M$ may be neglected. For simplicity, then, we have continued to use $m_{\mathrm{W}}=38.5 \mathrm{GeV} / \sin \theta_{\mathrm{W}}[8]$. 
TABLE 4

Two-loop results for the supersymmetric model with 4 generations as a function of the number of Higgs doublets $H$

\begin{tabular}{ccccccc}
\hline & $\begin{array}{c}\Delta \sin ^{2} \theta_{\mathrm{W}} \\
\left(10^{-3}\right)\end{array}$ & $\sin ^{2} \theta_{\mathrm{W}}$ & $\Delta \ln \left(M / m_{\mathrm{W}}\right)$ & $M\left(10^{14} \mathrm{GeV}\right)$ & $\Delta \alpha_{\mathrm{G}}^{-1}$ & $\alpha_{\mathrm{Gi}}^{-1}$ \\
\hline 0 & 3.1 & 0.206 & 0.274 & 12000 & -1.844 & 12.4 \\
2 & 3.7 & 0.234 & 0.161 & 250 & -1.694 & 11.9 \\
4 & 4.1 & 0.257 & 0.085 & 11 & -1.568 & 11.6 \\
6 & 4.4 & 0.276 & 0.032 & 0.81 & -1.461 & 11.3 \\
\hline
\end{tabular}

corrections are small, typically of order $+1 \%$ in $\sin ^{2} \theta_{\mathrm{w}}$ and of order $-25 \%$ in $M$, about half the corresponding changes due to the two-loop $\beta$-function in the standard model. As indicated at the close of the preceding section, effects due to the position of the supersymmetry threshold could be comparable. One-loop effects due to other particle thresholds either above or below the supersymmetry threshold could be even more significant. In addition, such a threshold leads to additional corrections at the two-loop level from dispersion in the masses about the threshold because of matching conditions similar to eq. (3.1). (See 3rd footnote of this section.)

One can also entertain the possibility of more than three matter generations. If a fourth generation occurs below $m_{\mathrm{W}}$, then the input values of $\alpha\left(m_{\mathrm{W}}\right)$ and $\alpha_{3}\left(m_{\mathrm{W}}\right)$ will be affected. Thus, even though at one-loop $\sin ^{2} \theta_{\mathrm{W}}^{(1)}$ and $t^{(1)}$ do not depend explicitly on $N_{\mathrm{g}}$, the predictions for $\theta_{\mathrm{w}}$ and $M$ will be changed because of these changes in the input coupling constants. Furthermore, the unified coupling constant $\alpha_{\mathrm{G}}$ depends explicitly on $N_{\mathrm{g}}$ already in the one-loop approximation. The two-loop corrections will then depend on $N_{\mathrm{g}}$ both implicitly through its dependence on $\sin ^{2} \theta_{\mathrm{W}}^{(1)}, t^{(1)}$, and $\alpha_{\mathrm{G}}^{(1)}$ and explicitly through the appearance of $N_{\mathrm{g}}$. [See eqs. (A.2) and (A.4).] Since we do not know whether there are more than three generations much less its mass scale, we will limit ourselves to calculating the effects of a fourth generation on $\alpha_{G}^{(1)}$ and on two-loop corrections, assuming the fourth generation has a mass scale of order $m_{\mathrm{W}}$. Such effects have been previously calculated in the standard model under similar assumptions [8,9]. In table 4, we display the effects of a fourth generation in the supersymmetric case. To one part in a thousand, the changes in $\sin ^{2} \theta_{\mathrm{W}}$ are the same as for $N_{\mathrm{g}}=3$. As for the unification mass, it is amusing to note that, while the two-loop corrections reduced $M$ for $N_{\mathrm{g}}=3$, they increase $M$ for $N_{\mathrm{g}}=4$.

\section{Summary and conclusions}

In this paper, we have analyzed several effects contributing to the evolution of the coupling constants of supersymmetric $\mathrm{SU}(3) \otimes \mathrm{SU}(2) \otimes \mathrm{U}(1)$, in both the one-loop and two-loop approximations. Because of the additional fields required by super- 
symmetry, unification is generally postponed compared to the standard model. However, this conclusion is sensitive to particle multiplets for which, when embedded in representations of the unified group, there is a large dispersion in the masses. The familiar example is the Weinberg-Salam Higgs doublet whose colored triplet partner composing the $\underset{\sim}{5}$ of $\mathrm{SU}(5)$ is presumed to have a mass of the order of the unification mass. As we have seen, however, a supersymmetric SU(5) model with four Higgs doublets (coming for example from a chiral and antichiral $\underset{\sim}{5}$ and 45 ) has a unification mass about the same as the minimal (non-supersymmetric) SU(5) model. However, $\sin ^{2} \theta_{\mathrm{w}}$ in such a case seems slightly too large $(\approx 0.25)$ compared to the currently favored experimental values. It seems that, if proton decay is observed, it will be difficult for supersymmetric $S U(5)$ to reconcile so short a lifetime with the experimental value of $\sin ^{2} \theta_{\mathrm{W}}$.

We also considered the effects of an intermediate threshold between $m_{\mathrm{W}}$ and $M$ at the one-loop level in a general way. We applied this to estimate the uncertainties in $\sin ^{2} \theta_{\mathrm{W}}, M$, and $\alpha_{\mathrm{G}}$ due to a threshold for supersymmetry breaking above $m_{\mathrm{W}}$. At the two-loop level, we have discussed the coupling constant evolution and the supersymmetric "matching conditions" near the unification mass between the three coupling constants of the $\mathrm{SU}(3) \otimes \mathrm{SU}(2) \otimes \mathrm{U}(1)$ effective gauge theory and the SU(5) unified coupling constant. Despite the many additional fields of supcrsymmetric SU(5), we found that the two loop corrections to the running coupling constants lead to even smaller corrections to $\sin ^{2} \theta_{\mathrm{W}}$ and to $M$ than in the minimal SU(5) model.

We would like to acknowledge many discussions with S. Dimopoulos. One of us (MBE) would like to thank M. Dine for several helpful conversations. We have enjoyed the hospitality of the Institute for Theoretical Physics, Santa Barbara, and of the Summer Institute on Grand Unification and Supersymmetry of the University of Washington, where this work was performed. This research has been supported in part by the US Department of Energy.

\section{Note added in proof}

Sakai [14] has presented arguments similar to those of ref. [4]. The sensitivity to the number of Higgs doublets noted herein has also been observed by Ibanez and Ross [15]. The uncertainty in the experimental determination of $\sin ^{2} \theta_{W}$ because of uncertainties in the value of $\rho=m_{\mathrm{W}}^{2} / m_{Z}^{2} \cos ^{2} \theta_{\mathrm{W}}$ has been emphasized in ref. [16].

It has recently been pointed out [17] that in SUMS there in general exist dimension five operators of the form $(1 / M) \bar{\psi} \psi \phi \phi$ which can also contribute to proton decay. Although further analysis [18] shows that the resulting decay rate (which depends on several unknown parameters such as the gluino mass) is not necessarily incompatible with experiments, it is clear that the relation between the unification mass $M$ and the proton decay rate is not so direct as we have implicitly assumed, unless the dimension five operators are eliminated by an additional symmetry. 


\section{Appendix}

The coefficients $b_{i}, b_{i j}$ for the $\beta$ function are defined by the perturbative expansion given in eq. (1.1). For the standard $S U(3) \otimes S U(2) \otimes U(1)$ model, they are given by the following:

$$
\begin{aligned}
& b_{i}=\left(\begin{array}{c}
0 \\
-\frac{22}{3} \\
-11
\end{array}\right)+N_{\mathrm{g}}\left(\begin{array}{c}
\frac{4}{3} \\
\frac{4}{3} \\
\frac{4}{3}
\end{array}\right)+H\left(\begin{array}{c}
\frac{1}{10} \\
\frac{1}{6} \\
0
\end{array}\right), \\
& b_{i j}=\left(\begin{array}{ccc}
0 & 0 & 0 \\
0 & -\frac{136}{3} & 0 \\
0 & 0 & -102
\end{array}\right)+N_{\mathrm{g}}\left(\begin{array}{ccc}
\frac{19}{15} & \frac{3}{5} & \frac{44}{15} \\
\frac{1}{5} & \frac{49}{3} & 4 \\
\frac{11}{30} & \frac{3}{2} & \frac{76}{3}
\end{array}\right)+H\left(\begin{array}{ccc}
\frac{9}{50} & \frac{9}{10} & 0 \\
\frac{3}{10} & \frac{13}{6} & 0 \\
0 & 0 & 0
\end{array}\right),
\end{aligned}
$$

where $N_{\mathrm{g}}$ is the number of matter generations and $H$ is the number of Higgs doublets.

For the supersymmetric extension of the standard model, it is a simple application of the formulas given in ref. [6] to obtain the coefficients. We find

$$
\begin{aligned}
& b_{i}=\left(\begin{array}{l}
0 \\
-6 \\
-9
\end{array}\right)+N_{\mathrm{g}}\left(\begin{array}{l}
2 \\
2 \\
2
\end{array}\right)+H\left(\begin{array}{c}
\frac{3}{10} \\
\frac{1}{2} \\
0
\end{array}\right), \\
& b_{i j}=\left(\begin{array}{rrr}
0 & 0 & 0 \\
0 & -24 & 0 \\
0 & 0 & -54
\end{array}\right)+N_{\mathrm{g}}\left(\begin{array}{ccc}
\frac{38}{15} & \frac{6}{5} & \frac{88}{15} \\
\frac{2}{5} & 14 & 8 \\
\frac{11}{15} & 3 & \frac{68}{3}
\end{array}\right)+H\left(\begin{array}{ccc}
\frac{9}{50} & \frac{9}{10} & 0 \\
\frac{3}{10} & \frac{7}{2} & 0 \\
0 & 0 & 0
\end{array}\right),
\end{aligned}
$$

where, of course, $N_{\mathrm{g}}$ is the number of generations of matter supermultiplets and $H$ is the number of Higgs doublet supermultiplets.

For convenience, we summarize the formulae for $\sin ^{2} \theta_{\mathrm{W}}, M$, and $\alpha_{\mathrm{G}}$ to two-loop order, adapting previous results [12] to the normalization conventions used here. Let us define a quantity $\Delta_{\imath}(\mu)$ by

$$
\frac{1}{\alpha_{i}(\mu)}=\frac{1}{\alpha_{\mathrm{G}}(\mu)}-\frac{\Delta_{i}(\mu)}{4 \pi},
$$

for $\mu$ in the neighborhood of the unification mass. The one-loop approximation $\Delta_{i}^{(1)}(\mu)$ is given in cq. (3.1), wherc, in the standard model with the modified minimal subtraction renormalization prescription, the constant $C_{i}$ is given in eq. (3.2) [most commonly without the second term]. In the supersymmetric case, we have argued the correct prescription for the constant is $C_{i}=0$. Let us denote the one-loop expressions defined in eq. (2.2) (leading to the values in table 2) as $\sin ^{2} \theta_{\mathrm{W}}^{(1)}, t^{(1)}$, and $\alpha_{\mathrm{G}}^{(1)}$. Then, in terms of the coefficients $b_{i}$ and $b_{i j}$ defined in eq. (1.11) [leading to eqs. (A.1) 
and (A.2)], the two-loop corrections can be written as

$$
\begin{aligned}
& \sin ^{2} \theta_{\mathrm{W}}^{(2)}=\sin ^{2} \theta_{\mathrm{W}}^{(1)}+\frac{C^{2} \alpha}{8 \pi d}\left\{\sum_{i j k} \varepsilon_{i j k}\left(b_{i}-b_{j}\right)\left[\Delta_{k}^{(1)}-\sum_{l} \frac{b_{k l}}{b_{l}} \ln X_{l}\right]\right\}, \\
& t^{(2)}=t^{(1)}+\frac{1}{4 \pi d}\left\{C^{2} \Delta_{1}^{(1)}+\Delta_{2}^{(1)}-\left(1+C^{2}\right) \Delta_{3}^{(1)}\right. \\
& \left.-\sum_{j}\left(C^{2} b_{1 j}+b_{2 j}-\left(1+C^{2}\right) b_{3 j}\right) \frac{\ln X_{j}}{b_{j}}\right\}, \\
& \frac{1}{\alpha_{\mathrm{G}}^{(2)}}=\frac{1}{\alpha_{\mathrm{G}}^{(1)}}+\frac{1}{4 \pi d}\left\{\begin{array}{cc}
C^{2} b_{1}+b_{2} & C^{2} \Delta_{1}+\Delta_{2} \\
b_{3} & \Delta_{3}
\end{array} \mid\right.
\end{aligned}
$$

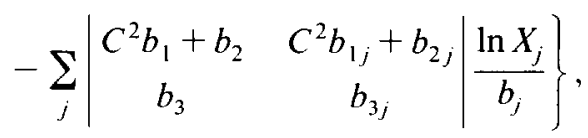

where the argument of the logarithm in each case is

$$
X_{j} \equiv 1+b_{j} \alpha_{\mathrm{G}}^{(1)} t^{(1)}
$$

With $C^{2}=\frac{5}{3}$ and $b_{i}, b_{i j}$ given by eqs. (A.1) or (A.2) above, it is now straightforward to compute the values given in table 3 .

\section{References}

[1] M. Veltman, Acta. Phys. Pol. B12 (1981) 437

[2] S. Dimopoulos and S. Raby, Nucl. Phys. B192 (1981) 353;

M. Dine, W. Fischler and M. Srednicki, Nucl. Phys. B189 (1981) 575:

E. Witten, Nucl. Phys. B185 (1981) 513

[3] S. Dimopoulos and H. Georgi, Nucl. Phys. B193 (1981) 150

[4] S. Dimopoulos, S. Raby and F. Wilczek, Phys. Rev. D24 (1981) 1681

[5] H. Georgi. H.R. Quinn and S. Weinberg. Phys. Rev. Lett. 33 (1974) 451

[6] D.R.T. Jones, Phys. Rev. D, to be published

[7] D.R.T. Jones, Nucl. Phys. B87 (1975) 127

[8] W.J. Marciano and A. Sirlin. Invited talk. Second Workshop on Grand unification, Ann Arbor. April, 1981

[9] J. Ellis, M.K. Gaillard, D.V. Nanopoulos and S. Rudaz, Nucl. Phys. B176 (1980) 61

[10] J. Ellis, 2 1st Scottish Universities Summer School, August, 1980, Ref. 2942-CERN (Sepl.. 1980)

[11] S. Dawson and H. Georgi, Phys. Rev. Lett. 43 (1979) 821

[12] T.J. Goldman and D.A. Ross, Nuc. Phys. B171 (1980) 273:

T. Hagiwara and N. Nakazawa. Phys. Rev. D23 (1981) 959

L.J. Hall. Nuct. Phys. B178 (1981) 75;

Y. Kazama, D.G. Unger and Y-P. Yao, UM HE 80-36 (1981):

Y. Kazama and Y.-P. Yao, Fermilab-Pub 81/18-THY (Jan. 1981);

C.H. Llewellyn-Smith, G.G. Ross and J.F. Wheater. Nucl. Phys. B177 (1981) 263; 
W.J. Marciano, Phys. Rev. D20 (1979) 274;

B.A. Ovrut and H.J. Schnitzer, Princeton Inst. for Advanced Study (March, 1981) and reference therein;

D.G. Unger and Y.-P. Yao, UM HE $81-30$ (1981);

S. Weinberg. Phys. Lett. 91B (1980) 51

[13] (a) W. Siegel, Phys. Lett. 84B (1979) 193

(b) D.M. Capper, D.R.T. Jones and P. van Nieuwenhuizen, Nucl. Phys. B167 (1980) 479;

W. Siegel, P.K. Townsend and P. van Nieuwenhuizen. Stony Brook ITP-SB-80-65 (January, 1981)

[14] N. Sakai, Tohuku University, TU/81/225

[15] L.E. Ibáñez and G.G. Ross, Oxford University ref. 65/81

[16] W.J. Marciano and G. Senjanovic, Brookhaven National Laboratory (Nov.. 1981)

[17] N. Sakai and T. Yanagida, Max-Planck-Institute MPI-PAE/PTh 55/81 (Oct., 1981):

S. Weinberg, Harvard University HUTP-81/A047

[18] S. Dimopoulos, S. Raby and F. Wilczek, University of Michigan UM HE 81-64 